\title{
Linx
}

Revue des linguistes de l'université Paris X Nanterre

$75 \mid 2017$

Imaginaires de la ponctuation. Ordre et inquiétude du discours

\section{Le point de suspension, idéogramme libertin du XVIII siècle}

Julien Rault

\section{CpenEdition}

Journals

Édition électronique

URL : http://journals.openedition.org/linx/1876

DOI : $10.4000 /$ linx.1876

ISSN : 2118-9692

Éditeur

Presses universitaires de Paris Nanterre

Édition imprimée

Date de publication : 22 décembre 2017

Pagination : $57-76$

ISBN : 978-2-84016-308-4

ISSN : 0246-8743

Référence électronique

Julien Rault, «Le point de suspension, idéogramme libertin du XVIIIe siècle », Linx [En ligne], 75 | 2017, mis en ligne le 23 novembre 2018, consulté le 20 avril 2019. URL : http://journals.openedition.org/ linx/1876 ; DOI : 10.4000/linx.1876

Ce document a été généré automatiquement le 20 avril 2019

Département de Sciences du langage, Université Paris Ouest 


\title{
Le point de suspension, idéogramme libertin du XVIII ${ }^{\mathrm{e}}$ siècle
}

\author{
Julien Rault
}

Règle $n^{\circ} 5$

Ah et tant qu'on y est, il reste une chose importante à savoir sur la ponctuation : les points de suspension ont toujours une valeur sexuelle. Toujours. Non, sérieusement : toujours. Si vous écrivez à votre collègue « Regarde tes mails, je viens de t'envoyer le fichier... », il traduira par « Je viens de t'envoyer le fichier et je tiens également à

t'informer que je ne porte pas de culotte».

(Grazia, « Les dix règles à respecter quand on envoie en SMS », mai 2012)

1 Les impératifs d'expressivité des nouvelles formes de communication écrite ont bouleversé les codes et les usages de la ponctuation. Le point, trop neutre, voire trop autoritaire ou trop disjoncteur, semble discrédité au profit des signes expressifs (!!!) et des émoticônes, qui tendent à se multiplier ${ }^{1}$. Parmi ces signes, le point de suspension possède un rôle tout à fait singulier : à l'intérieur du langage de l'immédiateté, de la concision et de la rapidité, l'introduction d'une altération en trois points, activant le virtuel et accordant, dans un espace réduit, une place à l'autre, ne peut être un événement dénué d'implications. La notion d'équivoque sexuelle, absolument récurrente, forme ainsi un aspect non-négligeable de l'imaginaire du signe au $\mathrm{XXI}^{\mathrm{e}}$ siècle. Le point de suspension, auquel nous attribuons le signifié minimal de latence ${ }^{2}$, se lit comme une réticence qui tend à devenir, comme l'atteste la déclaration péremptoire et humoristique du magazine cité en exergue, le plus souvent érotique, voire aphrodisiaque ${ }^{3}$.

La latence implique cependant beaucoup d'autres enjeux. La présence d'un signe qui dit sans dire, en apposant une persistance énonciative (un mi-dire), est nécessairement signifiante. En faisant apparaître que quelque chose est susceptible d'apparaitre, les trois points traduisent ainsi un je ne sais quoi qui échappe à la verbalisation, qui, à l'opposé du 
point, transgresse la clôture syntaxique et sémantique, produisant, sans aucun doute, une forme de dérèglement. L'imaginaire contemporain, évoquant la dimension séduisante de l'idéogramme ${ }^{4}$, s'appuie en réalité sur une conception beaucoup plus vaste, qui trouve son origine dans l'usage tout à fait singulier et novateur qu'en ont fait les libertins du XVIII ${ }^{\mathrm{e}}$ siècle.

Nous souhaitons montrer ici comment ce signe de ponctuation, simple convention typographique dans le théâtre français du XVII ${ }^{\mathrm{e}}$ siècle, a été investi et transformé par les différents courants libertins du XVIII ${ }^{e}$ siècle ${ }^{5}$; comment sur un plan diachronique, s'est constituée une dimension pérenne transgressive et satirique qui lie indissociablement l'imaginaire du signe à la pensée libertine. À une époque où la langue tend à se fixer, où le caractère clôturant du point se renforce, le développement d'un idéogramme du latent, qui indique l'affranchissement - premier sens du mot libertin -, se comprend comme un élément poétique et politique de dérèglement et de contestation. Depuis l'insertion fréquente dans les paroles rapportées, forme minime de transgression par rapport aux structures traditionnelles de l'écrit, jusqu'à sa prolifération inédite dans le récit satirique, au sein de fictions ménippéennes se confrontant aux limites, le ponctuant est l'instrument de la déstabilisation des structures linguistiques et littéraires (face à l'imaginaire de la langue classique) mais aussi sociales: l'altération de l'univocité du langage et la suspicion jetée sur la communication, produites par l'insertion d'un élément représentant le langage à l'état latent, forment des enjeux esthétiques autant qu'idéologiques et épistémologiques : la latence, disant l'excès et la labilité du sens et du sujet.

\section{Le contre-point}

Le point de suspension est l'un des signes de ponctuation apparu le plus tardivement, bien après la plupart des signes traditionnels. Si les marques d'ellipse, sous différentes formes, sont déjà présentes en Angleterre au XVI ${ }^{e}$ siècle (Henry, 2000, 2001), c'est au XVII ${ }^{e}$ siècle, dans le théâtre imprimé français (Riffaud, 2007), que la suite de points apparaît et se propage : ce ponctuant ${ }^{6}$ devient une convention permettant de signifier textuellement l'interruption d'un personnage et d'indiquer que la rupture syntaxique a été produite à dessein. Cet usage a connu un vif succès au point de véritablement influencer l'écriture dramaturgique $^{7}$. Ainsi, à la fin du XVII ${ }^{e}$ siècle, la suite de points indiquant une interruption ou une auto-interruption est parfaitement adoptée dans les pratiques scripturales, essentiellement théâtrales :

BAJAZET

[...] Je vous doy tout mon sang. Ma vie est vostre bien.

Mais enfin voulez-vous....

ROXANE

Non, je ne veux plus rien.

BAJAZET

Madame, ignorez-vous que l'orgüeil de l'Empire....

Que ne m'épargnez-vous la douleur de le dire! (Racine, $1672: 24,21$ )

5 À partir de cette fonction unique marquant l'interruption et l'inachèvement (que nous nommons suppression dans notre typologie ${ }^{8}$ ), il est possible d'envisager l'apparition 
ultérieure d'autres usages dans des phrases grammaticalement correctes et syntaxiquement achevées : suspension et supplémentation. De tels usages apparaissent et se développent réellement au XVIII ${ }^{\mathrm{e}}$ siècle et vont de pair avec l'introduction de ce ponctuant dans d'autres genres littéraires - notamment romanesques - mais aussi dans d'autres dispositifs énonciatifs - l'emploi réservé aux paroles glisse vers le récit. De la dénotation (objective) marquant l'interruption au théâtre, l'emploi des points multiples s'est doté de valeurs connotatives qui accroissent ses fonctions et donc ses implications énonciatives et sémantiques.

6 Les nouveaux usages, notamment en supplémentation, font apparaître dans le langage un affranchissement, un débordement, une contestation: l'achèvement syntaxique, la complétude sémantique, la possibilité d'un dire plein sont profondément remis en cause. Avant d'investir le champ littéraire, nous souhaitons au préalable formuler quelques hypothèses permettant de mieux comprendre l'apparition, et la prolifération, d'un tel élément de ponctuation, ainsi que son association étroite à la pensée libertine.

De façon significative, les nouveaux usages que nous venons de détailler coïncident avec un changement de statut du point et l'émergence de la notion de phrase. Au XVIII ${ }^{\mathrm{e}}$ siècle, l'idéologie prégnante, fondée sur l'idéal de l'achèvement et la « confiance en la perfection d'un langage » (Seguin, 1999a: 262), entraîne un accroissement du rôle clôturant du point : " on pourrait dire que le point libre a laissé la place à un point d'achèvement » (Seguin, 1999b : 5). C'est donc dans ce contexte particulier, parfaitement décrit par J.-P. Seguin, contexte néo-puriste qui cherche à fixer la ponctuation, que l'élément en plusieurs points se développe, apparaissant alors comme un véritable contre-point (syntaxique et donc idéologique). À mesure que disparaît «tout ce qui contredisait l'étanchéité absolue du point » (1999b:6), on constate la propagation d'un signe qui, disant la clôture impossible, correspond au besoin de produire le pendant négatif du point. Le potentiel subversif de cet élément de ponctuation est alors exhibé. Les nouvelles possibilités de fragmentation du discours offertes par le ponctuant permettent de transgresser une langue classique dont l'imaginaire est fondé sur la clarté, la logique et la raison; d'introduire également du mouvement et du désordre dans une langue "fixée ", « immobilisée » (Cohen, 1973 : 97), dont on a pu penser qu'elle était parvenue au point de perfection. Face à la rigidité et à l'absolutisme d'une époque où le «souci de l'ordre a envahi la grammaire » (Seguin, 1993 : 13), les points de latence font sans doute partie de «ces petites zones de fronde inconsciente » qu'évoquait J.-P. Seguin, "qui sont pour beaucoup dans le fameux "fossé" entre écrit et oral, discours spontané et discours surveillé, parole de prestige et parole libérée » (1999a : 304).

\section{Indicible (extase, censure)}

8 L'origine théâtrale du ponctuant le lie étroitement aux enjeux de l'oralité et par là, à ceux du corps et des affects. Le théâtre du XVIII siècle connaît ainsi, selon la formule de Dominique Maingueneau, une «inflation de points de suspension » (1986: 80), notamment dans le drame bourgeois théorisé par Diderot. Cette inflation, qui se comprend à l'aune du « débat sur l'expression linguistique de l'émotion » (1986: 81), est le reflet d'une volonté de déstabiliser la dimension continue et logique du langage. Il s'agit de se défaire du discours construit pour mieux suggérer les failles énonciatives liées aux soubresauts des affects.

DORVAL 
Rosalie,... si par malheur il étoit arrivé... que votre coeur surpris... fût entraîné par un penchant.... dont votre raison vous fît un crime... J'ai connu cet état cruel !... Que je vous plaindrois! (Diderot, $1757: 17$ )

En tant qu'opérateur syntaxique de déconstruction du langage savamment articulé et en tant que révélateur énonciatif de la présence d'un sujet défaillant, la suite de points est utilisée par Diderot (en suspension et en supplémentation) pour signifier les «cris», les «mots inarticulés», les "voix rompues», la «violence du sentiment coupant la respiration et portant le trouble dans l'esprit» (Diderot, 1757 : 1220). Elle constitue le médium pour imiter l'accent de la nature, soit le moment de lutte entre le langage et le silence, entre la voix et le corps. Les points de latence suggèrent ainsi l'inscription possible de la gestique là où la rigueur syntaxique de la tirade classique ne le permettait pas. Ils permettent de dire textuellement une présence, mutique, purement physique : le corps fait irruption dans le texte.

Du débordement du discours à l'excès sous toutes ses formes, le ponctuant se répand alors massivement dans des genres littéraires que l'on pourrait qualifier de satiriques, au sens large, de ménippéens, qui expérimentent et interrogent les limites :

Certains textes du XVIII ${ }^{e}$ siècle parmi lesquels avant tout les romans de Sade mais aussi, dans une autre mesure, ceux de Diderot, renouent avec un "genre » qu'on a $\mathrm{pu}$ appeler ménippée réalisé sur un double registre didactique et fictionnel (métalangue et littérature), reproduisant des expériences limites (aussi bien dans les comportements de sujets - sexualité, obscénité, etc. que dans leur langage qui s'écarte des normes de communication et se présente comme « jeu de mots » sinon comme « discours fou »); il procède par masque et par rire. (Kristeva, 1977 : 154)

11 Ce « langage » qui dit les comportements de l'excès, les « expériences limites », " discours fou » procédant "par masque », s'appuie de façon significative sur les points de latence, qui concentrent à eux seuls un nombre d'enjeux tout à fait emblématiques des préoccupations esthétiques, morales et politiques de telles œuvres. C'est pourquoi, dans la littérature libertine du XVIII ${ }^{\mathrm{e}}$ siècle (Crébillon, Rétif, Mirabeau et Sade), ils sont employés avec une grande fréquence ${ }^{9}$.

\begin{tabular}{|l|l|l|l|l|}
\hline Titres & Auteur & Année & Nombre de mots & $\ldots$ \\
\hline Le Sopha & Crébillon & 1742 & 87795 & 114 \\
\hline Les Bijoux indiscrets & Diderot & 1748 & 104912 & 423 \\
\hline Le Paysan perverti & Rétif & 1776 & 271306 & 1660 \\
\hline Le Libertin de qualité & Mirabeau & 1783 & 49199 & 951 \\
\hline Justine ou les malheurs de la vertu & Sade & 1791 & 138279 & 460 \\
\hline
\end{tabular}

12 À titre de comparaison, des romans célèbres comme Manon Lescaut (1731), La Vie de Marianne (1745) ou Paul et Virginie (1787) ne comportent, respectivement, que 6 occurrences pour $73499^{10}$ mots, 48 occurrences pour 246403 mots et 22 occurrences pour 46124 mots. Ce relevé, qui n'a certes pas la caution de l'exhaustivité, permet toutefois de se faire une idée de la présence massive du signe au sein d'un courant littéraire dont les ambitions esthétiques et idéologiques présentent un certain nombre de points de convergence. 
13 Une présence aussi importante de l'idéogramme se comprend tout d'abord par une radicalisation de l'usage « sensible » du signe. Le ponctuant inscrit textuellement l'extase qui vient prendre à défaut le langage :

La désarticulation du langage et la perte de conscience sont à l'horizon du plaisir.

La littérature libertine met en jeu le dérèglement de la langue au point où le sujet disparaît, où la raison balbutie.« Quel plai... ai... ai... ai... sir! ». Le mot se décompose. La musique sous la langue revient. La jouissance est là.

(Wald-Lakowski, 2011: 15)

14 Les points de latence interviennent afin de proposer un espacement signalant l'intervallement ${ }^{11}$ - dans lequel vient se loger le temps de la jouissance physique - qui sépare le discours de sa verbalisation. Dans l'extase, comme le dira plus tard Georges Bataille, « ce qui n'est pas utile doit se cacher (sous un masque)» (Bataille, 1970 : 196). Le masque est ici celui d'un ponctuant, assurant la pantomime du langage du corps entre les mots.

Ouf ! qu'est-ce là ? je suis.... je suis perdue,

Haï, haï, je n'en puis plus... Paix donc....

Cela me tue... (anonyme, $1787: 52$ )

Va, mon ami... va... foutre... Ah !... ah !.... Va fort !... Ah !... bougre !... Ah ! que tu fais

bien ça... Ah! ah! Ah !... (Mirabeau, 1783 : 938)

La poétique du corps subissant « la foudre du plaisir» (Nerciat, 1782: 709) passe par la fragmentation et se marque dorénavant par la suite de points (avec éventuellement la présence d'un autre signe expressif comme le point d'exclamation). Le discours articulé, savamment construit, se délite; le physiologique prend le pas sur le langage de la raison. La poétique se lie à la politique, réalisant un affranchissement dans la langue inhérent à la contestation libertine des valeurs et des règles établies. L'emploi d'un ponctuant, autorisant en outre toutes les libertés (syntaxiques) au XVIII ${ }^{\mathrm{e}}$ siècle, révèle un glissement très significatif, depuis l'usage originel, marquant la transgression de la clôture phrastique, jusqu'à la transgression généralisée.

La suite de points était à l'origine présente dans les cas de censure : ce type d'intervention contribue très certainement à renforcer l'idée de transgression. Ainsi, les premières occurrences de points multiples dans le récit se rencontrent au sein de textes libertins du XVII ${ }^{\mathrm{e}}$ siècle, en lieu et place des références bibliques : dans l'édition posthume de 1657 de l'Histoire comique de Cyrano de Bergerac, des termes ou des passages entiers, explicitement religieux, sont remplacés par une suite de points :

Par bonheur ce lieu estoit comme vous le sçaurez bien-tost. ....

... . Ainsi vous pouvez bien juger que sans ce hasard je serois mille fois mort.

(Cyrano de Bergerac, $1657: 29)^{12}$

Le ponctuant peut également se trouver adjoint à la première lettre de termes désignant de façon crue ou argotique les organes génitaux, ce qui l'associe alors étroitement au corps (sexué) :

Ces petits c.... dont l'on fait fête,

Où le v. ne met pas la tête,

N'assouvissent point mon désir :

J'aime les c... de belles marges,

Les grands c.... qui sont gros et larges

Où je m'enfonce à mon plaisir. (Anonyme, $1787: 54$ )

Ces occurrences, qu'elles soient le fait de la censure ecclésiastique ou plus largement morale, contribuent très certainement à façonner un imaginaire libertin du signe, en 
l'assimilant à l'idée d'affranchissement idéologique. En s'appuyant sur l'usage originel de la coupure, la littérature libertine du XVIII ${ }^{e}$ siècle, dans un mouvement de récupération ironique et subversif, a métamorphosé la ligne de points de censure en instrument de contestation. Sentant tout ce qu'elle pouvait tirer d'un ponctuant palimpseste, marqué au sceau du sacrilège, les romanciers libertins ont endossé ce signe de contrôle pour le retourner en signe contestataire, entre érotisation discursive (voiler pour mieux révéler) et décentrement idéologique (langage du corps).

Les points de suspension, depuis leur origine jusqu'au discours littéraire contemporain, entretiennent un lien privilégié avec la parole, avec le discours rapporté à la première personne, avec la fiction d'oralité. Ainsi, l'usage dans les paroles rapportées, conséquence directe de l'usage originel théâtral, est de loin le plus fréquent au XVIII ${ }^{e}$ siècle. Crébillon fils emploie la suite de points essentiellement dans les dialogues avec la fonction originelle d'interruption ${ }^{13}$, Diderot privilégie aussi les paroles rapportées, avec une tendance à la démarcation énonciative, Scarron également, mais de façon plus économe. Rares sont les auteurs qui usent du ponctuant en dehors des paroles rapportées. Rétif est l'un de ceux-là; accompagnant souvent le pathos (les larmes), participant du style «sensible»:

Enfin il aperçut son père, appuyé contre un jeune arbre planté par Edmond luimême, une main sur son front, de l'autre essuyant quelques larmes...

[...] heureusement ses larmes coulèrent, elles inondèrent le fils méritant qu'elle pressait contre son sein... (Restif de la Bretonne, $1779: 21,58$ )

Dans un autre roman épistolaire, Laclos fait également intervenir la suite de points, avec une fréquence notable, dans le mécanisme de séduction et de frustration lié au temps différé de la réception. Celle-ci permet alors de créer un espace intersubjectif qui exemplifie la distance qui sépare le scripteur du lecteur pour mieux l'abolir, en jouant sur la connivence ou l'adresse :

J'en suis dans une fureur..... Eh bien ? vous ne devinez pas encore ? Oh! l'esprit lourd!

Oh ! je la troublerai..... Promettez-moi que je la troublerai. (Choderlos de Laclos, $1782: 25,82)$

21 Les points multiples participent du jeu de l'attente, qui est un jeu de dupe ; ils séduisent au sens où ils conduisent à l'émetteur et illustrent ainsi la manipulation à l'œuvre. Ces emplois émergents dans le récit semblent particulièrement significatifs, dans la mesure où leurs enjeux se distinguent radicalement des précédents : il ne s'agit plus de mimer une oralité fictive. Le transfert du ponctuant de la parole vers le récit, véritablement inauguré au XVIII siècle, a inversé le positionnement énonciatif, porté dorénavant du côté de la suggestion :

Après que je fus baigné, épongé, séché, la maîtresse de maison m'oignit avec une liqueur qui me rendit la peau douce comme du satin ; me coupa les cheveux, quand je dis les cheveux..... (Anonyme, $1787: 18$ ) Introduite dans le récit, la suite de points devient le signe d'un sujet non plus dépossédé de son discours mais au contraire le maîtrisant pleinement. Ce glissement de la dépossession à la maîtrise illustre l'infléchissement des usages (de la suppression à la supplémentation) et oriente nettement les enjeux du côté la domination. 


\section{De la dépossession à la domination : instruments sadiens}

23 S'il existe dorénavant de véritables études stylistiques sur l'œuvre de Sade, jamais le rôle de la ponctuation n'a été envisagé. Or l'usage du Marquis, au regard de celui de ses contemporains, n'est absolument pas conventionnel. Alors qu'il ne se préoccupe absolument pas de ponctuation, notamment du point, quasi inexistant dans les manuscrits, Sade apporte un soin particulier à l'introduction d'un double ou triple point qui apparaît comme un prolongement sémantique tout à fait emblématique d'une nouvelle posture énonciative. Introduisant avec une fréquence tout à fait inédite le ponctuant dans le récit - a fortiori au passé et à la troisième personne, le Marquis de Sade fait véritablement figure de précurseur :

[...] et pour l'instant, le cœur, la richesse et la confiance de $m$ de corville conseiller d'etat, homme dans le plus grand crédit et a la veille d'entrer dans le ministere .. la route fut epineuse, .. on n'en doute assurément pas, c'est par l'apprentissage le plus honteux et le plus dur, que ces demoiselles la font leur chemin [...].

Ce fut ici ou la malheureuse Juliette oubliant tous les sentiments de sa naissance, et de sa bonne education pervertie par de mauvais livres et de mauvais conseils, pressée de jouir seul, d'avoir un nom, et point de chaîne, osa se livrer à la coupable pensée d'abreuger les jours de son mari .. (Sade, $1787: 6,9$ )

L'emploi singulier - et ce, comme le révèle le manuscrit, jusque dans la forme graphique du ponctuant auquel il attribue tantôt deux, tantôt trois points, non sans une certaine cohérence $^{14}$ - induit un changement significatif, comme l'atteste cette occurrence du signe double encadrant le syntagme ".. la route fut épineuse ..." ou encore celle prolongeant « la coupable pensée » (« d'abreuger les jours de son mari .. »). L'évolution du libertinage sous l'influence sadienne se comprend aussi à la lumière de l'évolution des emplois de la suite de points : l'auteur des Infortunes de la vertu, amenant à son paroxysme la perversion dans le courant libertin aux dépens de la séduction, introduit un usage qui ne sert plus uniquement à traduire les affres de la passion et le langage désarticulé sous le coup d'une dépossession de soi (usages traditionnels chez Diderot, comme nous l'avons $\mathrm{vu}$ ), mais permet de signifier la parfaite maitrise de l'instance énonciative, la domination totale : dès lors l'intervention des points multiples peut concerner aussi bien les victimes (dépossédées) que les libertins et le narrateur lui-même (dominants). Un tel emploi est parfaitement révélateur de ce courant littéraire apparu vers 1750 et qui se pose contre le sentimentalisme,

[...] comme un jeu de société aristocratique où rien n'est aussi compromettant que l'amour-passion, élément irrationnel dans un jeu intellectuel où s'exprime le souci de l'amour-propre et de la domination de l'autre autant que celui du plaisir physique et dont l'œuvre de Sade constitue l'apogée [...]. (Peeters, 2002 : 330)

La suite de points devient, par un glissement vers la connotation, un élément du récit qui instaure un surplomb et une distance. La supplémentation sadienne, tout en pastichant le style sensible, organise le glissement du pathétique au cynique, de la dépossession à la domination, exhibant un sujet de l'énonciation maître de son discours.

[...] ils y mettoient enfin toutes les delicatesses quil etoit possible d'attendre de deux ames sensibles..

[...] deja les traces de l'infortune s'effacoit du front charmant de l'aimable justine .. deja les graces y retablissoient leur empire [...] (Sade, $1787: 135)$ 
de points participe alors, pour reprendre l'expression de Marcel Hénaff, d'une forme « d'insistance oblique » (1978: 16). Une correction apportée dans le manuscrit de la première version de Justine, avec l'ajout suscrit du signe, dit également assez bien les fonctions qui sont conférées à la suspension :

l'objet essentiel est de ne jamais rien refuser et de tout prevenir

l'objet essentiel est de ne jamais leur rien refuser ... de les prevenir surtout (Sade, $1787: 78)$

L'insertion du point de latence en lieu et place de la coordination va dans le sens d'un écartèlement de l'organisation phrastique mais aussi d'un renforcement, d'une exacerbation sadique que l'ajout de l'adverbe "surtout » a posteriori vient confirmer. L'intervalle créé par les trois points instaure graphiquement un contre-point à l'assertion qui précède («[...] ce soin rempli, les orgies commencent, te les detailler seroit impossible, daussi bizarres caprices peuvent ils jamais etre reglés [...]») : l'ajout de l'élément dans un second temps illustre la volonté de produire un espace dans lequel le détail des sévices, refusé explicitement auparavant, pourra prendre place dans l'esprit du lecteur. La saturation sadienne - catalyse analysée par Barthes (1971) - passe aussi, et paradoxalement, par ces espaces d'évitement du langage ; après avoir épuisé le dire (le tout dire), le signe permet de signifier qu'il est encore possible de dire (autrement).

L'idéogramme du latent vient s'ajouter au tout dire libertin : sursignifiant, il est instrumentalisé pour produire l'effroi. Soit la scène vide produite par les points sera évoquée par la suite avec force détails (auquel cas l'élément ponctuant, jouant de la prétérition, participe évidemment de la saturation), soit, dans de plus rares cas, elle ne l'est pas et l'espace en points multiples devient alors ce lieu emblématique où toute verbalisation est impossible. Si tout est dit, jusqu'à l'extrême violence, qu'y a-t-il derrière ces points? Ni elliptiques, ni catalytiques, les points de latence participent de la saturation de l'espace et créent une surenchère en exacerbant l'horreur, de l'ordre de l'excès absolu, indicible, « seulement imaginable à la limite des mots » (Hénaff, 1978 : 93). Un phénomène significatif qui rejoint, sur un plan phrastique, ce que disait notamment Michel Foucault :

Sade parvient au bout du discours et de la pensée classiques. Il règne exactement à leur limite. (1966: 224)

L'examen des deux versions ultérieures ${ }^{15} \mathrm{du}$ conte révèle des changements majeurs et significatifs dans l'emploi des points multiples. La majorité des occurrences précédemment citées a disparu et d'autres font leur apparition :

Justine en larmes va trouver son curé; elle lui peint son état avec l'énergique candeur de son âge... Elle était en petit fourreau blanc. (Sade, 1791 : 135)

[...] douée d'une tendresse, d'une sensibilité surprenante, au lieu de l'art et de la finesse de son aînée, elle n'avait qu'une ingénuité... une candeur, qui devait la faire tomber dans bien des pièges. (Sade, $1799: 397$ )

Ces deux occurrences sont assez représentatives de l'évolution des emplois. Les points interviennent désormais à la suite d'une évocation des traits de caractère des protagonistes, afin de mieux ironiser sur le poncif de la " candeur » ou de l'« ingénuité », tout en soulignant le contraste et la dimension perfide du " piège » qui ne manque pas de se refermer sur les plus innocents. Le mouvement d'une Justine à l'autre est bien, comme l'écrit Michel Gailliard, celui d'« une plongée progressive dans le sadisme» $(2006: 44)$ et ce mouvement se traduit aussi par l'emploi d'un élément de ponctuation désormais aux 
deux extrémités, sans cesse du côté de l'excès (de cruauté, de candeur) : l'exacerbation de l'antithèse est très nette dans la troisième version :

si Justine échappait à un danger en se débarrassant de la Dubois et de ses compagnons, ce n'était que pour tomber peut-être dans un plus réel, en se livrant à son cher oncle... Oh Dieu! Après d'aussi grands services !...

Ici l'égarement du libertin était à son comble : ses fougueuses passions venaient de briser tous les freins...

[...] sur ce corps inanimé... sans défense (Sade, 1799 : 461, 464, 466)

$C^{\prime}$ 'est donc très certainement à Sade qu'il faut en revenir pour interroger et comprendre certains emplois tout à fait novateurs. Ce dernier, qui effectue un usage fréquent et singulier du ponctuant dans le récit, est sensible en outre à la dimension physiologique du signe de ponctuation dans l'écriture. On note par exemple l'intervention récurrente de la suite de points en présence du sème du «frémissement » (en amont ou en aval) : «[...] dont l'abord me fit frémir bien plus quil ne me consola ... un tremblement involontaire me saisit alors [...] », «antonin s'approche de moi .. je fremis, [...]» $(1787: 63,82)$. Les points de latence sadiens, lorsqu'ils ne viennent pas souligner le pathos et l'horreur, semblent ménager un espace de frémissement (« la peau du texte » frémit à l'unisson de celle du corps $\left.{ }^{16}\right)$. Et deviennent ainsi proprement «signifiants», si l'on s'en tient à l'acception donnée par Roland Barthes à ce mot: «c'est le sens en ce qu'il est produit sensuellement » (1973: 82).

A n'en pas douter, les points multiples sont l'un des instruments du rituel sadique, participant de la suspicion et de la séduction. Leurs interventions régulières dessinent une succession de paliers qui ouvrent chaque fois sur "l'abîme " ${ }^{17}$. Il suffit pour s'en convaincre de se reporter à la fréquence, au sein de la même œuvre (Les Infortunes de la vertu, 1787), de l'interjection « hélas » suivant immédiatement l'intervention des points de latence (sur 7 occurrences de l'interjection dans l'œuvre, 5 sont précédées d'une suite de points : « jecrivis une lettre aussi touchante que je le pus .. helas elle ne l'etoit que trop [...] », $1787: 52)$. Stigmates de l'expérience douloureuse, le ponctuant, en deux ou trois points, introduit dans la linéarité le cahot d'un cheminement déceptif, matérialisant le processus de déniaisement face au réel, telles les étapes successives d'une atroce et inexorable catabase.

\section{Prolongement}

La suite de points est un ponctuant intrinsèquement libertin, au sens plein, et doit sans conteste être inscrite dans "l'isotopie libertine» (Gailliard, 2006: 1999). De la parole rapportée au récit, du discours "gazé » (allusif, de Crébillon, par exemple ${ }^{18}$ ) au discours total marqué par la volonté de tout dire (« l'encyclopédie de l'excès » ${ }^{19}$ sadienne), les points multiples se lisent comme la conséquence d'une volonté de transcription de tout ce qui traverse - le corps - et excède - le langage verbalisé.

À partir de cette dimension, fondamentalement libertine, qui fait du point de suspension un signe litote, trace ténue d'un dire total, il est possible de mieux comprendre les représentations contemporaines associées au signe, notamment dans les nouveaux moyens de communication (texto, courriel). Le point de suspension, signe séducteur (conduire à soi), signe de la séduction, lié à l'imaginaire libertin, se pare de vertus érotisantes. Nous avons vu que nombreux éléments permettent de comprendre cette 
association, depuis les phénomènes de censure morale jusqu'aux problématiques du nondit et de la réticence.

Les humoristes, qui font leur miel des nouveaux usages apparus dans les différents supports de communication contemporains, appréhendent le point de suspension de façon relativement similaire. Gad Elmaleh ${ }^{20}$ évoque par exemple les avantages des messages textos qui permettent «de séduire plus facilement » et dans lesquels on peut même « installer de l'ambiguïté grâce à trois points de suspension » : signe de l'ambiguïté, de l'équivoque, le point de suspension est du côté de la séduction : "c'est comme si tu disais : on sait jamais qu'est-ce qu'y va se passer ». Une scène du film Intouchables reprend également cette idée de séduction, en éradiquant cette fois - ce qui est le support du comique - toute forme d'ambiguïté. À propos du message envoyé au personnage joué par François Cluzet par une femme qu'il souhaite séduire, Driss, le personnage joué par Omar Sy, est catégorique :

Driss : Elle a écrit: "Je viens à Paris la semaine prochaine, appelle-moi...", trois petits points. Vous comprenez ce que ça veut dire?

Philippe : C'est bon, ça?

Driss : Bien sûr que c'est bon, trois petits points! un point, deux points, trois points : trois points, elle veut pécho! ${ }^{21}$

Dans une série humoristique au format très court (Bref.), diffusée sur Canal $+^{22}$, des auteurs mettent en scène un personnage devant envoyer un texto à une femme rencontrée quelques heures plus tôt. Après de multiples tergiversations sur la formulation idoine, se pose la question de l'achèvement: «pour donner un côté "j'en pense plus que je n'en dis", j'ai voulu mettre trois petits points»; le personnage se ravise cependant, considérant que cela fait « un peu pervers ». Il met alors un point, qu'il trouve aussitôt « un peu autoritaire» et finit par mettre deux points horizontaux au lieu de trois, estimant l'équivoque moins marquée.

37 Au-delà du rapport particulier - affectif - au signifiant (le signifiant semble lui-même séduisant : «trois petits points ", « un point, deux points, trois points »), attestant même d'une réelle réflexion sur les enjeux de la forme graphique (un point étant trop " autoritaire", trois points "trop pervers»), on constate un glissement relativement significatif de l'implicite à l'explicite. Le prolongement en trois points qui permettait de marquer « l'ambiguïté » devient un élément absolument univoque (« elle veut pécho»), au point d'être perçu comme une forme de déviance au regard de la bienséance morale et sociale («un peu pervers»). Le signe, employé dans le langage texto, est bien l'idéogramme langagier de la sexualité : la valeur de latence, expressive, à défaut d'être encore transgressive, suppose désormais une attention particulière portée à la dimension érotique : dire en-deçà, dire au-delà, par le biais d'un signe dont Walter Benjamin disait déjà, au début du XXe siècle, qu'il était un code universel (Morse) en littérature pour représenter le langage de l'amour ${ }^{23}$. 


\section{BIBLIOGRAPHIE}

\section{Euvres}

ANONYME, 1787, Le Lutteur, ou le Petit-fils d'Hercule, reproduit dans Taylor institution, University of Oxford, 1992.

CHODERLOS DE LACLOS, P.-A.-F., 1782, Les Liaisons dangereuses, ou Lettres recueillies dans une société \& publiées pour l'instruction de quelques autres, Paris, Durand Neveu.

CREBILLON FILS, 1736, Les Égarements du cour et de l'esprit, Paris, Garnier Flammarion, 1985

CYRANO DE BERGERAC, S., 1657, Histoire comique : contenant les états et empires de la Lune, Paris, Charles de Sercy.

DIDEROT, D., 1757, Le Fils naturel, ou les épreuves de le vertu, Amsterdam, M. M. Rey.

DIDEROT, D., 1757, Entretiens sur le fils naturel, Cuvres, Paris, Gallimard, Pléiade, 1975.

MIRABEAU, H.-G., 1783, « Ma conversion », Romanciers libertins du XVIII ${ }^{e}$ siècle, tome 2, WaldLasowski, P. (dir.), Paris, Gallimard, Pléiade, 2005.

NERCIAT, 1782, Féliciou ou mes fredaines II, Romanciers libertins du XVIII ${ }^{e}$ siècle, tome 2, WaldLasowski, P. (dir.), Paris, Gallimard, Pléiade, 2005.

RACINE, J., 1672, Bajazet, Acte II, scène 1, Paris, chez Pierre Le Monnier.

RESTIF DE LA BRETONNE, E., 1779, La Vie de mon père, Paris, Garnier Frères, 1970.

SADE, D.-A.-F., 1787, Les Infortunes de la vertu, Présentation, transcription et notes par JeanChristophe Abramovici, Paris, Zulma, Bibliothèque Nationale de France, CNRS éditions, coll. « Manuscrits », 1995.

SADE, D.-A.-F., 1791, Justine ou Les Malheurs de la vertu, tome 2, Paris, Gallimard, Pléiade, 1995.

SADE, D.-A.-F., 1799, La Nouvelle Justine ou Les Malheurs de la vertu, tome 2, Paris, Gallimard, Pléiade, 1995.

\section{Travaux critiques}

BARTHES, R., 1971, Sade, Fourier, Loyola, Paris, Seuil.

BATAILLE, G., 1970, « Méthode de méditation », Euvres complètes, tome 5, Paris, Gallimard.

BENJAMIN, W., 1927-1930, Selected writings, Jennings M., Eiland H., Smith G. (éd.), vol. 2, part. 1, First Harvard University Press, 2005.

BENVENISTE, E., 1974, « Sémiologie de la langue », Problèmes de linguistique générale, tome II, Paris, Gallimard.

BIKIALO, S., RAULT, J., 2016, « Ponctuation, rythme et espace graphique », La Ponctuation à l'aube du XXI e siècle : perspectives historiques et usages contemporains, Pétillon S., Rinck F., Gautier A. (dir.), Lambert Lucas, p. 175-198.

BRUNET, E., 1999, « La langue française au XXe siècle. Ce que disent les chiffres », Nouvelle Histoire de la langue française, J. Chaurand (dir.), Paris, Seuil.

COHEN, M., 1973, Histoire d'une langue : le français, Paris, Editions Sociales. 
DELON, M., 2000, Le Savoir-vivre libertin, Paris, Hachette Littératures, coll. « Pluriel ».

DÜRRENMATT, J., 1998, Bien coupé, mal cousu. De la ponctuation et de la division du texte romantique, Paris, PUV, coll. « Essais et savoirs ».

DÜRRENMATT, J., 2015, La Ponctuation en français, Ophrys.

FOUCAULT, M., 1966, Les Mots et les choses, Paris, Gallimard.

GAILLIARD, M., 2006, Le Langage de l'obscénité. Étude stylistique des romans de Sade : Les Cent Vingt Journées de Sodome, les trois Justine et Histoire de Juliette, Paris, Honoré Champion, Bibliothèque de grammaire et de linguistique.

HAWCROFT, M., 2012, «Punctuating dramatic dialogue : Corneille's suspension points », dans Modern Language Review, vol. 107, part. 1, p. 136-155.

HENAFF, M., 1978, Sade. L'invention du corps libertin, Paris, PUF, coll. « Croisées ».

HENRY, A., 2000, In Ellipsis... : The History of Suspension Marks in British Literature with Particular Reference to the Eighteenth- and Nineteenth-Century Novel, (thèse de doctorat non-publiée, University of Cambridge.

HENRY, A., 2001, « Ellipsis Marks in a Historical Perspective », dans The Motivated Sign: Iconicity, Language and Literature, ${ }^{\circ} 2$, Olga Fischer and Max Nänny, (éds.), John Benjamins, p. 135-155.

KLIEBENSTEIN, G., 2004, « Quos ego... mais... un... un... Remarques sur l'hémiphasie », dans La Réticence, La Licorne, n68, Louvel L., Rannoux C. (dir.), Poitiers, MSHS, p. 135-151.

KRISTEVA, J., 1977, « La musique parlée ou remarques sur la subjectivité dans la fiction, à propos du « Neveu de Rameau », dans Langue et langages de Leibniz à l'Encyclopédie, Duchet M., Jalley M., (dir.), Séminaire de l'École normale supérieure de Fontenay, 10/18, p. 153-206

LABORDE, A.-M., 1974, Sade romancier, La Baconnière.

LE BRUN, A., 1993, Soudain un bloc d'abîme. Sade, Paris, Gallimard.

MAINGUENEAU, D., 1986, « Le langage en suspens », Paroles inachevées, DRLAV n³4-35, p. 77-94.

MESCHONNIC, H., 1982, Critique du rythme. Anthropologie historique du langage, Paris, Verdier.

RAULT, J., 2014, Poétique du point de suspension. Valeur et interprétations, Thèse de doctorat soutenue à l'Université de Poitiers.

RAULT, J., 2015, Poétique du point de suspension. Essai sur le signe du latent, Nantes, Cécile Defaut.

RAULT J., 2016, «"Espacements" et "effets" de lecture : la suite de points », La Ponctuation à l'aube du XXI e siècle : perspectives historiques et usages contemporains, Pétillon S., Rinck F., Gautier A. (dir.), Lambert Lucas, p. 139-148.

RIFFAUD, A., 2007, La Ponctuation dans le théâtre imprimé au XVIIème siècle, Chap. VII, « La typographie au service du théâtre : l'invention des points de suspension », Genève, DROZ.

SEGUIN, J-P., 1993, L'Invention de la phrase au XVIII siècle, Bibliothèque de l'Information Grammaticale, Bruxelles, Peeters publishers.

SEGUIN, J.-P., 1999a, " La langue française aux XVII ${ }^{e}$ et XVIII ${ }^{e}$ siècles ", dans Nouvelle Histoire de la langue française, Chaurand J. (dir.), Paris, Seuil.

SEGUIN, J.-P., 1999b, «Éléments pour une stylistique de la phrase dans la langue littéraire du XVIII ${ }^{\mathrm{e}}$ siècle ", dans L'Information grammaticale, $\mathrm{n}^{\circ}$ 82, p. 5-15;

WALD LASOWSKI, P., 2008, Le Grand dérèglement, Paris, Gallimard, coll. « L'infini ». 
WALD LASOWSKI, P., 2011, Dictionnaire libertin, Paris, Gallimard.

\section{NOTES}

1. Voir notamment le chapitre 5 du dernier ouvrage de Jacques Dürrenmatt (2015).

2. La notion de « latence » permet de définir un signe qui se refuse à la saisie définitionnelle : ce dernier est le plus souvent appréhendé par le concept, trop étroit, de suspension, ou bien par le biais d'une adjonction de termes antithétiques, relevant le plus souvent de différents niveaux de l'analyse (interruption et prolongement, lacune et ajout, présence et absence, etc.) : la valeur de latence tient compte de la polyvalence du signe et embrasse la pluralité des interprétations discursives (voir Rault : 2014 et 2015).

3. Sur la dimension aphrodisiaque de la réticence, voir notamment Kliebenstein (2004:139).

4. Bien qu'il soit capable d'endosser une multitude d'interprétations, ou plutôt, parce qu'il est capable d'endosser une multitude d'interprétations, le point de suspension peut être envisagé comme un idéogramme dans la mesure où nous considérons que chaque usage se fonde a minima sur le signifié de latence (faire apparaître que quelque chose est susceptible d'apparaître).

5. Les relevés d'Étienne Brunet (1999: 720) sur le corpus Frantext confirment le développement des usages: la progression est constante jusqu'en 1780 où apparaît nettement un point culminant, précédant une éclipse relative avant la reprise et la progression importante dès 1830 .

6. Dans la mesure où le signifiant n'est pas stabilisé, la forme graphique pouvant aller de deux à huit points, l'emploi du mot «signe » peut paraître impropre. S'il nous arrive de l'employer, du fait de son large spectre sémantique et dans un souci de variation, nous privilégions les termes "ponctuant», «points multiples» ou «suite de points", considérant que l'emploi du terme « signe » ne sera judicieux qu'avec la stabilisation du signifiant en trois points, laquelle a lieu au cours de la deuxième moitié du XIX ${ }^{\mathrm{e}}$ siècle.

7. «But as the use of suspension points for this purpose became conventional, there is some evidence that Corneille started to incorporate more suspended discourse " (Hawcroft, 2012 : 131). L'introduction des points multiples aurait ainsi eu une influence sur l'écriture, et plus largement sur la représentation des échanges dans le dialogue cornélien : dans les éditions ultérieures, comme l'a bien montré Mickael Hawcroft, Corneille revisite ses dialogues et réécrit plusieurs répliques en ajoutant des coupures supplémentaires (Mélite en comporte 15 en 1633 et 38 en 1663).

8. Nous synthétisons ici notre proposition (Rault, 2015 : 55-62) de typologie fondée sur une tripartition représentative des trois principaux usages et enjeux, sur un plan diachronique et synchronique, de la valeur de latence :

1. Suppression (latence = non-réalisation in esse . Lacune) : La Marquise sortit à...

2. Suspension (latence $=$ réalisation in fieri. Attente) $:$ La Marquise sortit... à cinq heures.

3. Supplémentation (latence $=$ réalisation in posse. Ajout) $:$ La Marquise sortit à cinq heures...

9. Le relevé qui suit s'appuie sur la base de données Frantext.

10. Une fois déduites les occurrences (abondantes) des points suivant la majuscule d'un nom propre.

11. Nous reprenons ici la distinction opérée entre espacement (organisation spatiale du mouvement) et intervallement (organisation temporelle du mouvement) par Bikialo et Rault (2016).

12. La version initiale étant : «Par bonheur, ce lieu était comme vous le saurez bientôt, le Paradis terrestre, et l'arbre sur lequel je tombai se trouva justement l'Arbre de vie. Ainsi vous pouvez bien juger que sans ce hasard je serois mille fois mort. »

13. «Je me souviens... Madame, interrompit-il, en poussant cela [...]», 
"Si vous le vouliez même, continuai-je... Non, assurément, interrompit-elle, je ne veux rien. ", Crébillon fils, Les Égarements du cour et de l'esprit, (1736), Garnier Flammarion, 1985, p. 191, p. 205. 14. Sans qu'il y ait à proprement parler de régularité dans l'usage sadien, on constate cependant une tendance à introduire deux points dans le récit et trois points dans le discours rapporté. Une occurrence de cinq points apparaît au cours d'un échange : " pourriés vous mexpliquer ..... non c'est la notre secret» (Ibid., p. 85). La présence d'un nombre de points plus élevé dans un usage prototypique - puisqu'il y a vraisemblablement interruption ou effet d'interruption ici - laisse penser que la réduction du nombre à deux ou trois points du signifiant graphique accompagne bien d'autres enjeux. Par ailleurs, l'emploi de la forme en deux ou trois points, prolongeant un énoncé complet, indique à elle seule un changement important: la réduction de la propriété matérielle de l'élément accompagne le nouvel usage, dans la mesure où la forme graphique la plus répandue au XVIII était encore la suite de quatre points, marquant l'interruption syntaxique et sémantique. L'infléchissement discursif produit par certains auteurs se double d'un infléchissement formel, graphique.

15. Les versions ultérieures se caractérisent en outre, comme l'a bien montré A.-M. Laborde, par des effets de mouvement, de dynamisme et d'accélération, liés à l'emploi du présent, du style direct et à l'utilisation de phrases courtes (1974:51).

16. Pour Marcel Hénaff, au contraire, «La peau du texte, comme celle du corps, est sans frémissement émotionnel, sans lapsus, sans signification cachée » $(1978: 63)$.

17. Voir par exemple Annie Le Brun, Soudain un bloc d'abîme. Sade, Gallimard, 1993.

18. Delon M., (2000 : 37).

19. Hénaff M., (1978), p. 65.

20. Elmaleh G., Papa est en haut, spectacle mis en scène par Judith Elmaleh, 2007-2008.

21. Intouchables, réalisé par Olivier Nakache et Éric Toledano, 2011.

22. Khojandi K., Bref., « Bref, j'ai envoyé un texto », épisode 77, juillet 2012.

23. " "..." and " - ", the universal Morse code used in literature to represent the language of love " (Benjamin W., "A state monopoly on pornography", Walter Benjamin, Selected writings, Jennings M., Eiland H., Smith G. (éd.), vol. 2, part. 1, 1927-1930, First Harvard University Press, 2005 , p. 72

\section{RÉSUMÉS}

Cet article souhaite montrer comment le point de suspension, dont l'usage s'est véritablement développé au XVIII ${ }^{\mathrm{e}}$ siècle, est absolument emblématique des enjeux esthétiques, idéologiques et épistémologiques de la pensée libertine ; comment, sur un plan diachronique, s'est constituée une dimension pérenne transgressive et satirique du signe de ponctuation au regard d'un imaginaire de la langue classique, fondé sur la clarté, l'hermétisme, l'achèvement. Le XVIII ${ }^{\mathrm{e}}$ siècle apparaît ainsi comme le moment déterminant dans la constitution d'un imaginaire libertin du point de suspension, que l'on peut comprendre à partir de la valeur de latence, laquelle introduit, face au point de l'ordre, l'excès et la labilité du sens et du sujet. Un contre-point.

This work considers the suspension point, whose uses really developed in the XVIIIth century, as an element absolutely symbolic of the esthetical, ideological and epistemological stakes in the libertine thought ; on a diachronic plan, this punctuation mark is transgressive and satiric compared to an imagination of the classic language, based on clarity, hermetism, completion. 
The XVIIIth century so appears as a crucial moment in the constitution of a libertine imagination of a punctuation mark whose value can be defined with the term of latency.

\section{AUTEUR}

\section{JULIEN RAULT}

Université de Poitiers, EA 3816 